\title{
Nomogram for estimating specific consanguinity risks
}

\author{
RICARDO CRUZ-COKE
}

From the Genetic Unit, Hospital JJ Aguirre, and the Faculty of Medicine, University of Chile, Santiago, Chile

SUMMARY This paper describes a nomogram to estimate the chance of consanguinity for specific autosomal recessive diseases, taking into account the gene frequencies $(q)$ of the recessive alleles and the coefficient of inbreeding (F) of the family of the proband.

Consanguinity is an important problem in medical genetics because it significantly influences morbidity risks for recessive disorders. Genetic counsellors manage this problem in very simple terms according to Mendelian rules but without considering the theory of inbreeding in full. Consequently, the severity of specific recessive genetic risks is not accurately estimated. The purpose of this paper is to set out a nomogram to solve some consanguinity problems in cases of autosomal recessive disorders.

A nomogram is a graphical representation of numerical relations that enables the value of a dependent variable to be read off when the value of two or more independent variables are given. The figure shows a nomogram plotted on a logarithmic scale depicting the classical variables of the theory of inbreeding according to the equations given by $\mathrm{Li}^{12}$ This nomogram presents the frequency of inbred homozygous recessive subjects $\left(\mathbf{R}_{\mathbf{F}}=\right.$ $\mathrm{q}^{2}+\mathrm{Fpq}$ ) as a function of the ratio of inbred homozygous $\left(\mathbf{R}_{F}\right)$ to panmictic homozygous subjects $\left(R_{0}=q^{2}\right)$ for different values of inbreeding coefficients (F) and gene frequencies (q) of recessive alleles. The relative consanguinity risk defined as $\mathrm{q}^{2}+\mathrm{Fpq} / \mathrm{q}^{2}$ may be computed also as $1+\mathrm{F} / \mathrm{q}$ when $\mathrm{q}$ is small.

This nomogram illustrates in full the three basic properties of a system of inbreeding that may be useful in accurately estimating consanguinity risks in man.

(1) Coefficient $F$ measures the degree of increase of homozygosity and decrease of heterozygosity, and consequently represents the degree of fixation of the recessive allele $q$. The lines of $F$ are plotted converging to the point of fixation, where all the variables merge $\left(F, q, R_{F}=1\right)$.

(2) In a system of inbreeding the gene frequencies (q) are independent of the inbreeding coefficient (F). The nomogram shows that increasing $F$ does not change gene frequencies, which are plotted perpendicularly to the $F$ lines.

(3) The proportion of recessives may be divided

Received for publication 20 August 1981

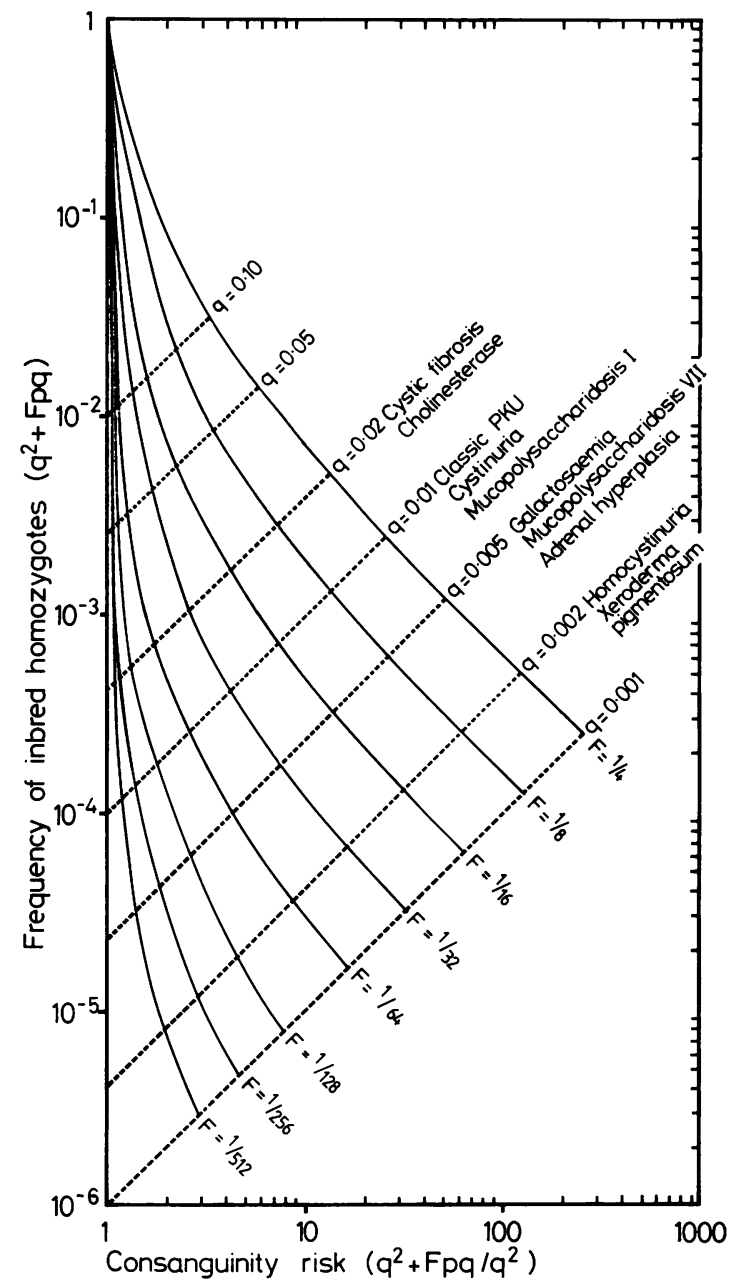

FIGURE Nomogram for estimating consanguinity risks according to gene frequencies $(q)$ and inbreeding coefficients $(F)$. Classical recessive diseases with their estimated mean gene frequencies for European populations are given (data from Stanbury et al ${ }^{\mathbf{3}}$ ). 
into $R_{F}$ with inbreeding and $R_{0}$ without inbreeding (ratio $R_{F} / R_{0}$ ) which shows that the consanguinity risk is greater than unity. For any given value of $F$, the ratio increases with the decrease of gene frequencies. The lowest is the recessive allele and the highest is the risk of a child being homozygous.

In order to estimate specific consanguinity risks, it is necessary to take into account the gene frequencies of recessive disorders at given $F$ values. When $\mathrm{q}$ and $\mathrm{F}$ are known it is very easy to compute these risks using the equation $1+F / q$. Nevertheless the advantage of this nomogram is that it is possible to read off the risks for most common classical recessive disorders extended in a wide range of gene frequencies from 0.02 to 0.002 . It is possible to compare risks with equal $F$ and different $q$ values, and risks with equal $\mathrm{q}$ and different $\mathrm{F}$ values.
Moreover, this nomogram provides a useful diagram to give an overview of the situation and an explanation of the magnitude of the genetic risks involved.

\section{References}

1 Li CC. Population genetics, Chicago: Chicago University Press, 1955.

$2 \mathrm{Li}$ CC. First course in population genetics, California: Pacific Grove, 1976.

3 Stanbury JB. Wyngaarden JB, Frederickson DS. The metabolic basis of inherited diseases. 4th ed. New York: McGraw-Hill, 1978.

Requests for reprints to Professor R Cruz-Coke, Genetic Unit, Hospital J J Aguirre, Santiago 7, Chile. 